\title{
How low do we fall to lower hemoglobin A1c? SGLT2 inhibitors: Effective drugs or expensive toxins!
}

\author{
Udaya M. Kabadi ${ }^{1,2,3}$ \\ ${ }^{1}$ Broadlawns Medical Center, Des Moines, USA; ukabadi@gmail.com \\ ${ }^{2}$ Des Moines University, Des Moines, USA \\ ${ }^{3}$ University of Iowa, Iowa City, USA
}

Received 14 July 2013; revised 12 August 2013; accepted 20 August 2013

Copyright (C) 2013 Udaya M. Kabadi. This is an open access article distributed under the Creative Commons Attribution License, which permits unrestricted use, distribution, and reproduction in any medium, provided the original work is properly cited.

\begin{abstract}
SGLT2 inhibitors improve hyperglycemia with a decline in body weight and blood pressure, the outcomes touted to be of major importance in management of type 2 diabetes. However, the efficacy in lowering glycemia is very modest and imparts an undue cost burden on patients. Moreover, the declines in body weight and blood pressure are slight at best and orthostatic hypotension and its manifestations including syncope at worst, are all secondary to dehydration and probable total body electrolyte depletion caused by polyuria and nocturia as anticipated and established consequences of glycolsuria induced by SGLT2 inhibitors via their mechanism of action. Moreover, frequent adverse effects including impaired renal function as expressed by rises in serum creatinine and urea nitrogen, hyperkalemia and recurrent genitourinary infections are certainly likely to lead to the decline in quality of life. Finally, a rise in LDL cholesterol accompanied by increased serum viscosity secondary to dehydration may compromise cardiovascular safety as well. Thus, use of these expensive agents with modest efficacy and with established significant pathologic side effects escalating costs even further and unknown long term safety may be contradictory to the tenets of ethical medical practice established by "Hippocratic oath". Recent approval of SGLT2 inhibitors by regulatory agencies in Europe and USA for treatment of hyperglycemia in type 2 diabetes is therefore surprising and disheartening.
\end{abstract}

Keywords: Type 2 Diabetes; SGL2 Inhibitor;

Dehydration; Genitourinary Infection

\section{INTRODUCTION}

As a member, it was shocking and surprising to see an advertisement sticker for "Invokana" on the front cover of "Diabetes Care" as well as "Journal of Clin Endo Metab". What is it that American Diabetes Association and The Endocrine Society are intending and attempting to "Invoke"? All throughout my medical training, as a student, a resident in internal medicine and then as fellow in endocrinology, I was taught and trained by my preceptors to reverse and prevent glycosuria and therefore, loss of fluid and various electrolytes in order to relieve symptoms, e.g. polyuria, polydypsia, nocturia, as well as the other consequential clinical manifestations of dehydration, e.g. dizziness, fatigue, muscle cramps, lethargy etc. Moreover, reversal of glycosuria also induced remission and prevented recurrences of genitourinary infections. Thus, the aim of treatment was also to improve the quality of life by providing relief of symptoms.

\section{DISCUSSION}

The proponents of SGLT2 inhibitors promote the "beneficial" effects of weight loss, lowering of blood pressure and a decline in HbA1c. These effects are exaggerated when compared to placebo rather than with the baseline values [1-8]. I must point out that both the weight loss and lowering of blood pressure are secondary to dehydration as documented by rises in both serum creatinine and urea nitrogen concentrations and therefore may not be necessarily healthy. Dehydration is certainly induced by polyuria or "pollakiuria" caused by glycosuria and/or genitourinary tract infections. The clinical trials failed to assess total body losses of electrolytes which are almost certainly likely to occur with polyuria although serum electrolytes may not significantly alter in presence of normal renal function. Nevertheless, hyperkalemia has been observed in subjects with renal dysfunction as well with concurrent administration of drugs 
inhibiting renin-angiotensin-aldosterone system even in presence of normal renal function [1-8]. And al be it, these are the drugs most frequently recommended in subjects with type 2 diabetes for treatment of hypertension and for preservation of renal function. Moreover, "pollakiuria" is already frequently present in both elderly men secondary to prostatism and in several post menopausal women, the populations most predisposed to presence of type 2 diabetes. Fortunately, a caution is issued regarding occurrence of "orthostasis" in the elderly as a result of dehydration as well as increased prevalence of genitourinary infections in all comers [1-13]. However, the long term consequences of persistent exposure of urinary tract including urinary bladder to hyperglycemic hypertonic urine are unknown although increased risk of bladder cancer has been described [14]. Moreover, the data regarding negative impact of polyuria and/or "pollakiuria" on "quality of life", e.g. recurrent visits to toilets with urgency and precipitancy during wakefulness and even incontinence during sleep resulting in sleeplessness and its consequences although well known in clinical practice, are not assessed in the clinical trials using these drugs [1-13]. Finally, a rise in serum LDL cholesterol and non HDL cholesterol levels as well as increased serum viscosity secondary to dehydration noted in clinical trials are known to induce and promote occurrence of hypercoagulable milieu responsible for ensuing thrombotic adverse cardiovascular outcomes, e.g. deep vein thrombosis, myocardial ischemia, stroke etc. [1-13]. The concern regarding long term safety of these drugs has been raised recently in an extensive review and meta analyses since the relative risk for occurrence of non fatal strokes is documented to be significantly higher in subjects receiving canagliflozin when compared with subjects administered placebo $[15,16]$. In actuality, the natural product "phlorizin" from which these drugs have evolved was once considered a "nephrotoxin" because it induced glycosuria and its consequences, e.g. dehydration, electrolyte depletion and genito urinary infections [17]. Therefore, it is rather unfortunate to note that regulatory agencies, especially FDA did not require studies examining cardiovascular safety of Canagliflozin prior to approval against its own mandate especially in the light of its recent decision to recommend a long term clinical trial to establish cardiovascular safety of another drug e.g. "degludec" prior to approval. Moreover, the cost efficacy analysis of these drugs for lowering HbAlc and for treatment of side effects is likely to show it in "poor" light even in the short term. Finally, let's not forget what even the modern Hippocratic Oath states "I will apply, for the benefit of the sick, all measures which are required, avoiding those twin traps of overtreatment and therapeutic nihilism", and thus, relieve (not worsen) symptoms and most importantly "Do No Harm" [18].

\section{CONCLUSION}

SGLT2 inhibitors are very modest in their efficacy in terms of lowering glycemia while incurring undue cost burden to the patients with a mechanism of action detrimental to quality of life attributed to polyuria, nocturia and consequential dehydration including orthostatic hypotension. Moreover, electrolyte imbalance, declining renal function as evident by rises in serum creatinine and urea nitrogen concentrations as well as recurrent genitourinary infections and occurrence of ketonemia and ketonuria are likely to further compromise the quality of life. Finally, questions regarding the increase in prevalence of bladder and breast cancers and long term cardiovascular safety of SGLT2 inhibitors need definitive resolution. Thus, these agents are neither cost effective nor safe in the short term and the long term efficacy and safety remains to be established. Therefore, recent approved entry of SGL2 inhibitors into the drug paraphernalia for treatment of type 2 diabetes by regulatory agencies is apparently a downward stumble rather than progress.

\section{REFERENCES}

[1] Rosenstock, J., Aggarwal, N., Polidori, D., et al. (2012) Dose-ranging effects of canagliflozin, a sodium-glucose cotransporter 2 inhibitor, as add-on to metformin in subjects with type 2 diabetes. Diabetes Care, 35, 1232-1238. http://dx.doi.org/10.2337/dc11-1926

[2] Stenlöf, K., Cefalu, W., Kim, K., et al. (2013) Efficacy and safety of canagliflozin monotherapy in subjects with type 2 diabetes mellitus inadequately controlled with diet and exercise. Diabetes, Obesity and Metabolism, 4, 372382. http://dx.doi.org/10.1111/dom. 12054

[3] Cefalu, W., Leiter, L., Yoon, K., et al. (2013) Efficacy and safety of canagliflozin versus glimepiride in patients with type 2 diabetes inadequately controlled with metformin (CANTATA-SU): 52 week resultsfrom a randomised, double-blind, phase 3 non-inferiority trial. Lancet, 382, 941950.

[4] Schernthaner, G., Gross, J.L., Rosenstock, J., et al. (2013) Canagliflozin compared with sitagliptin for patients with type 2 diabetes who do not have adequate glycemic control with metformin plus sulfonylurea: A 52-week randomized trial. Diabetes Care, 36, 2508-2515.

[5] Gerich, J.E. and Bastien, A. (2011) Development of the sodium-glucose co-transporter inhibitor dapagliflozin for the treatment of patients with type 2 diabetes mellitus. Expert Review of Clinical Pharmacology, 4, 669-683. http://dx.doi.org/10.1586/ecp.11.54

[6] Wilding, J.P., Woo, V., Rohwedder, K., et al. (2013) Dapagliflozin in patients with type 2 diabetes receiving high doses of insulin: Efficacy and safety over two years. Diabetes, Obesity and Metabolism.

[7] Bolinder, J., Ljunggren, O., Johansson, L., et al. (2013) Dapagliflozin maintains glycaemic control while reduc- 
ing weight and body fat mass over 2 years in patients with type 2 diabetes mellitus inadequately controlled on metformin. Diabetes, Obesity and Metabolism. http://dx.doi.org/10.1111/dom.12189

[8] Bailey, C.J., Gross, J.L., Hennicken, D., et al. (2013) Dapagliflozin add-on to metformin in type 2 diabetes inadequately controlled with metformin: A randomized, double-blind, placebo-controlled 102-week trial. BMC Medicine, 11, 43.

http://dx.doi.org/10.1186/1741-7015-11-43

[9] Lamos, E.M., Younk, L.M. and Davis, S.N. (2013) Canagliflozin, an inhibitor of sodium-glucose cotransporter 2, for the treatment of type 2 diabetes mellitus. Expert Opinion on Drug Metabolism \& Toxicology, 9, 763-775. http://dx.doi.org/10.1517/17425255.2013.791282

[10] Nyirjesy, P., Zhao, Y., Ways, K., et al. (2012) Evaluation of vulvovaginal symptoms and Candida colonization in women with type 2 diabetes mellitus treated with canagliflozin, a sodium glucose co-transporter 2 inhibitor. Current Medical Research and Opinion, 28, 1173-1178. http://dx.doi.org/10.1185/03007995.2012.697053

[11] Nicolle, L.E., Capuano, G., Ways, K., et al. (2012) Effect of canagliflozin, a sodium glucose co-transporter 2 (SGLT2) inhibitor, on bacteriuria and urinary tract infection in subjects with type 2 diabetes enrolled in a 12-week, phase 2 study. Current Medical Research and Opinion, 28, $1167-1171$.

http://dx.doi.org/10.1185/03007995.2012.689956
[12] Johnsson, K.M., Ptaszynska, A., Schmitz, B., et al. (2013) Urinary tract infections in patients with diabetes treated with dapagliflozin. Journal of Diabetes and Its Complications, 27, 473-478.

[13] Johnsson, K.M., Ptaszynska, A., Schmitz, B., et al. (2013) Vulvovaginitis and balanitis in patients with diabetes treated with dapagliflozin. Journal of Diabetes and Its Complications, 27, 479-484.

[14] Clar, C., Gill, J.A., Court, R., et al. (2012) Systematic review of SGLT2 receptor inhibitors in dual or triple therapy in type 2 diabetes. BMJ Open, 2, e001007.

[15] Riser, T. and Harris, K. (2013) The clinical efficacy and safety of sodium glucose cotransporter-2 inhibitors in adults with type 2 diabetes mellitus. Pharmacotherapy, 33, 984-999.

[16] Vasilakou, D., Karagiannis, T., Athenasiadou, E., et al. (2013) Sodium-glucose cotransporter 2 inhibitors for type 2 diabetes. Annals of Internal Medicine, 159, 262-274. http://dx.doi.org/10.7326/0003-4819-159-4-201308200-0 $\underline{0007}$

[17] Ehrenkranz, J.R., Lewis, N.G., Kahn, C.R. and Roth, J. (2005) Phlorizin: A review. Diabetes/Metabolism Research and Reviews, 21, 31-38. http://dx.doi.org/10.1002/dmrr.532

[18] Medicine Net.com (2013) Definition of hippocratic oath. Newsletter, 24 May. 